\title{
Edge Extraction Based on Aperture Synthesis in Optical Scanning Holography
}

\author{
Haiyan Ou, ${ }^{1, *}$ Edmund Y. Lam, ${ }^{2}$ and Bing-Zhong Wang ${ }^{1}$ \\ ${ }^{1}$ Institute of Applied Physics, University of Electronic Science and Technology of China, 610054, Chengdu, China \\ ${ }^{2}$ Department of Electrical and Electronic Engineering, University of Hong Kong, Pokfulam, Hong Kong \\ *Corresponding author: ouhaiyan@uestc.edu.cn
}

\begin{abstract}
We present an edge extraction method based on aperture synthesis with different pupils in optical scanning holography. By utilizing two sub-holograms covering different spatial frequency ranges of the object, sharp edges can be extracted successfully.
\end{abstract}

OCIS codes: $090.1995,100.3190,100.3010,110.1758$

\section{Introduction}

Optical scanning holography (OSH) is an incoherent digital holography technique, with wide applications such as optical microscopy, remote sensing etc [1]. It uses a Fresnel zone plate (FZP) to record the volume information of an object by two-dimensional (2D) raster scanning. For optical image processing, it is highly desirable that the edge information can be extracted via reconstruction. Various techniques have been proposed to meet this requirement, including ways of manipulating the pupil functions [2,3], or algorithm such as total variation regularization with a nonnegative constraint [4].

In this paper, we propose an edge extraction method based on aperture synthesis, which is an effective solution for obtaining superresolution [5,6]. In the recording stage, two sub-holograms covering different spatial frequency ranges from the object are generated by manipulating the location of the pupil function. With the higher and lower spatial frequency signals, an edge extraction as well as an enhanced edge contrast in sectioning can be realized.

\section{Principle}

The schematic of the OSH system with the proposed approach is illustrated in Fig. 1. A laser beam centered at $\omega$ is divided into two parts by the beamsplitter BS1. One of the branch would pass through a pupil with function $p_{1}(x, y)=1$ to have a spherical wavefront; the other part would first go through a frequency shift $\Omega$ via the acounsto-optic frequency shifter (AOFS) and then pass through the second pupil with function $p_{2}(x, y)$. BS2 is used to combine the two parts together. The Fresnel zone plate (FZP) generated by different wavefront is then used to scan the object, which is a distance $z$ away from the scanning mirror. The light from the object is collected by lens 3 and converted to electrical signal via the photodiode (PD). The digital hologram is then generated after some electrical processing [1].

In the recording stage, two sub-holograms would be generated with different pupil functions $p_{2 a}(x, y)=\delta(x-$ $\left.\Delta x_{1}, y-\Delta y_{1}\right)$ and $p_{2 b}(x, y)=\delta\left(x-\Delta x_{2}, y-\Delta y_{2}\right)$, where $\Delta x_{*}$ and $\Delta y_{*}$ denote the shift in the $x$-axis or $y$-axis.

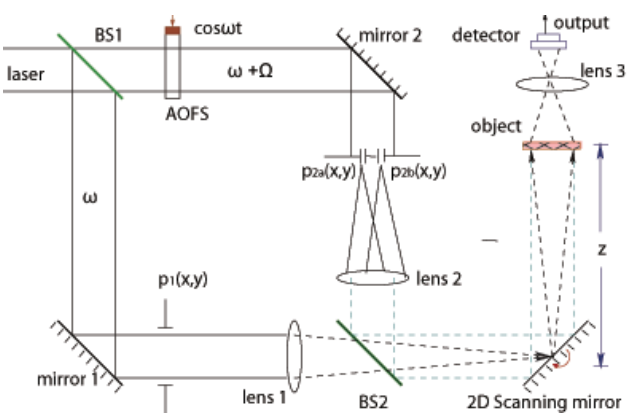

Fig. 1. Schematic of the OSH system. 
For the first measurement, the impulse response of the OSH system can be expressed as

$$
h_{1}(x, y ; z)=j \frac{1}{\lambda z} \exp \left\{j \frac{\pi}{\lambda z}\left(\left(x-\Delta x_{1}\right)^{2}+\left(y-\Delta y_{1}\right)^{2}\right)\right\},
$$

where $x, y, z$ are the spatial coordinates, and $\lambda$ is the wavelength of the optical source.

Suppose an object with $n$ sections are scanned. Then the generated sub-hologram $g_{1}$ can be expressed as

$$
g_{1}(x, y)=\sum_{i=1}^{n} \varphi\left(x, y ; z_{i}\right) * h_{1}\left(x, y ; z_{i}\right)
$$

where $z_{i}$ and $\varphi\left(x, y, z_{i}\right)$ represent the depth location and the intensity distribution of the $i$-th section, respectively.

For the second measurement, a different sub-hologram $g_{2}(x, y)$ generated by the impulse response $h_{2}(x, y ; z)$ can also be expected. Other than merging the sub-holograms with their relative positions and phase in traditional aperture synthesis technique $[5,6]$, we combined them together to retrieve the edge information. The combined hologram can be expressed in matrix form as

$$
\mathbf{g}=\left[\begin{array}{l}
\mathbf{g}_{1} \\
\mathbf{g}_{2}
\end{array}\right]=\left[\begin{array}{llll}
\mathrm{H}_{1}\left(z_{1}\right) & \mathrm{H}_{1}\left(z_{2}\right) & \ldots & \mathrm{H}_{1}\left(z_{n}\right) \\
\mathrm{H}_{2}\left(z_{1}\right) & \mathrm{H}_{2}\left(z_{2}\right) & \ldots & \mathrm{H}_{2}\left(z_{n}\right)
\end{array}\right]\left[\begin{array}{c}
\boldsymbol{\varphi}_{1} \\
\boldsymbol{\varphi}_{2} \\
\vdots \\
\boldsymbol{\varphi}_{n}
\end{array}\right]+\left[\begin{array}{c}
\boldsymbol{n}_{1} \\
\boldsymbol{n}_{2} \\
\vdots \\
\boldsymbol{n}_{n}
\end{array}\right]=\mathrm{H} \boldsymbol{\varphi}+\boldsymbol{n},
$$

where $n$ denotes the noise, $\varphi$ is the object at various sections, and $\mathrm{H}$ represents the combined matrices of the first and second measurement. Traditional method for sectioning convolves the hologram with the conjugated FZPs, which suffers from large defocus noise and blurred edge [1]. Here, we regarded it as a minimization problem

$$
\boldsymbol{\varphi}_{\mathrm{est}}=\arg \min _{\boldsymbol{\varphi}}\|\mathrm{H} \boldsymbol{\varphi}-\mathbf{g}\|_{2}^{2}+\rho\|\mathrm{C} \boldsymbol{\varphi}\|_{2}^{2},
$$

where $\|\cdot\|_{2}$ denotes the $\ell_{2}$ norm, $\rho>0$ is the regularization parameter, and $C$ matrix stands for the Laplacian of Gaussian, which is essentially a high-pass filter. Noted that the second term of Eq. (4) is the edge of the object, if $\boldsymbol{\varphi}$ is the solution [7]. This indicates that the edge information can be extracted with the sub-holograms. The minimization problem can be solved by using conjugate gradient method, the detail of which can be found in [8].

\section{Simulation and analysis}

The proposed method is demonstrated via simulation in this section. The laser source with wavelength equal to $632 \mathrm{~nm}$ is used. The diameter of the collimated beam is $D=40 \mathrm{~mm}$, and the focal length of lens 1 and lens 2 is $f=50 \mathrm{~mm}$. In the recording stage, $p_{2}$ is first set as $p_{2 a}(x, y)=\delta\left(x-\Delta x_{1}, y-\Delta y_{1}\right)$, with $\Delta x_{1}=\Delta y_{1}=0$, and then switch to $p_{2 b}(x, y)=\delta\left(x-\Delta x_{2}, y-\Delta y_{2}\right)$, with $\Delta x_{2}=0.3 \mathrm{~mm}$ and $\Delta y_{2}=0 \mathrm{~mm}$. The generated FZPs located at $z=34 \mathrm{~mm}$ are shown in Fig. 2. One can see from Fig. 2 (a) and (b) that the opaque and transparent zones of the FZP pattern would change with the pupil function $p_{2}$, indicating that different spatial frequency ranges from the object would be recorded.

We first demonstrate the case with an object of single section, which is $1 \mathrm{~mm} \times 1 \mathrm{~mm}$ in size, and is sampled to $512 \times 512$ pixels, as is shown in Fig. 3(a). The reconstructed sectional image with traditional method as well as the proposed one are shown in Fig. 3(b) and (c), in which the latter shows better edge contrast. The extracted edge is shown in Fig. 3(d). One can see from this figure that the edge information has been extracted successfully.

The case for an object with two sections are also analyzed, with section distance equal to $1 \mu \mathrm{m}$. The simulation results are shown in Fig. 4. As can be seen from Fig. 4 that the proposed method also outperforms the traditional one in preserving the edge. The edge information of each section can also be extracted with small defocus noise.

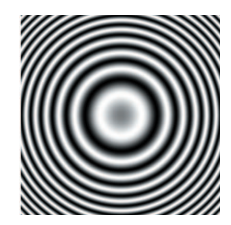

(a) $p_{2 a}(x, y)=\delta\left(x-\Delta x_{1}, y-\Delta y_{1}\right)$

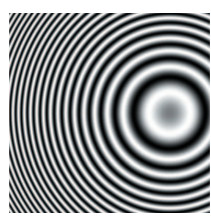

(b) $p_{2 b}(x, y)=\delta\left(x-\Delta x_{2}, y-\Delta y_{2}\right)$

Fig. 2. The FZPs at $z=34 \mathrm{~mm}$ with $\Delta x_{1}=\Delta y_{1}=0 \mathrm{~mm}, \Delta x_{2}=0.3 \mathrm{~mm}$, and $\Delta y_{2}=0 \mathrm{~mm}$. 


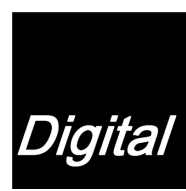

(a)

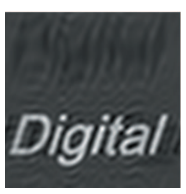

(b)

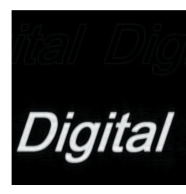

(c)

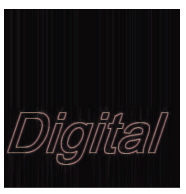

(d)

Fig. 3. Case for single section: (a) the object, (b) reconstructed section with conventional method, (c) reconstructed section with the proposed method, and (d) the extracted edge of the object.

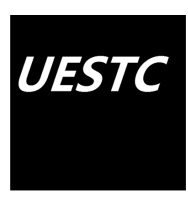

(a)

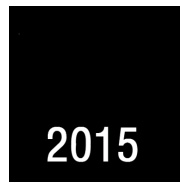

(e)

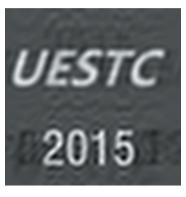

(b)

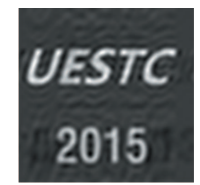

(f)

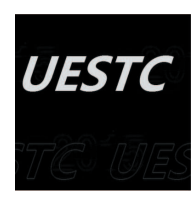

(c)

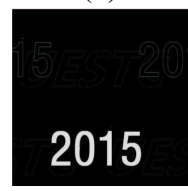

(g)

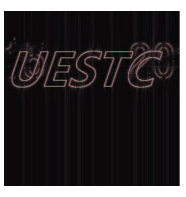

(d)

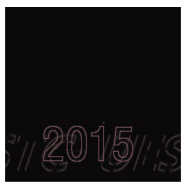

(h)

Fig. 4. Case for two sections: section 1 (a) and section 2 (e), reconstruction with conventional method for section 1 (b) and section 2 (f), reconstruction with the proposed method for section 1 (c) and section $2(\mathrm{~g})$, the extracted edge of section 1 (d) and section $2(\mathrm{~h})$.

\section{Conclusion}

We propose an edge extraction method in OSH based on aperture synthesis. By recording two subholograms covering different spatial frequency range from the object, an enhanced edge contrast can be realized, and the edge information can be extracted as well.

\section{Acknowledgement}

This research was supported in part by the National Science Foundation of China (Grants 61361166008), Hong Kong Research Grants Council (HKU 7131/12E), and by NSFC/RGC (N-HKU714/13).

\section{References}

1. T.-C. Poon, Optical Scanning Holography with MATLAB (Springer, 2007).

2. Y. Pan, W. Jia, J. Yu, K. Dobson, C. Zhou, Y. Wang, and T.-C. Poon, "Edge extraction using a time-varying vortex beam in incoherent digital holography," Opt. Lett. 39, 4176-4179 (2014).

3. X. Zhang and E. Lam, "Edge detection of three-dimensional objects by manipulating pupil functions in an optical scanning holography system," in "IEEE International Conference on Image Processing," (2010), pp. 3661-3664.

4. X. Zhang and E. Y. Lam, "Edge-preserving sectional image reconstruction in optical scanning holography," J. Opt. Soc. Am. A 27, 1630-1637 (2010).

5. R. Binet, J. Colineau, and J. Lehureau, "Short-range synthetic aperture imaging at $633 \mathrm{~nm}$ by digital holography," Appl. Opt. 41, 4775-4782 (2002).

6. C. Yuan, H. Zhai, and H. Liu, "Angular multiplexing in pulsed digital holography for aperture synthesis," Opt. Lett. 33, 2356-2358 (2008).

7. D. Marr and E. Hildreth, "Theory of edge detection," Proc. R. Soc. Lond. B 41, 187-217 (1980).

8. H. Ou, T.-C. Poon, K. K. Wong, and E. Y. Lam, "Depth resolution enhancement in double-detection optical scanning holography," Appl. Opt. 52, 3079-3087 (2013). 